\title{
ÜBER ACHTSAMKEIT UND SELFCARE IN BESONDEREN ZEITEN ...
}

\section{Liebe Kolleginnen und Kollegen!}

Der Herbst läutet traditionell ein neues Ausbildungsjahr ein, mit frischem Elan nach der Sommerpause geht's ans Unterrichten, Weiterbilden und Vorbereiten.

Eigentlich - denn heuer ist ja alles anders.

So eine richtige Sommerpause gab es nicht, waren doch viele Dinge, die coronabedingt verkompliziert wurden oder verschoben werden mussten, abzuarbeiten bzw. vorzubereiten. Das gilt für die OGKA als Verein, aber wahrscheinlich auch für viele KollegInnen im Einzelnen.

Wir müssen unsere Ressourcen als ÄrztInnen und als Privatpersonen heuer besonders gut im Auge behalten, gerade eben, weil uns möglicherweise Durchschnauf- und Batterieaufladezeiten fehlen. Wir können unseren helfenden und heilenden Beruf nur ausüben, wenn wir selbst gesund sind, gerade in der Akupunktur arbeiten wir mit Energie. Deshalb ist Achtsamkeit uns selbst gegenüber eine unserer wichtigsten Verantwortungen, auch unseren Patienten gegenüber. Generell und in besonders anspruchsvollen Zeiten wie diesen umso mehr. Akupunktieren Sie sich selbst oder gönnen Sie sich eine Behandlung bei einem Kollegen, oft vergessen wir, uns selbst das zu gönnen, was wir an Stärkung und Harmonisierung unseren Patienten tagtäglich zukommen lassen. Wir haben ein wunderbares Werkzeug zur Gesunderhaltung und Gesundung, über das wir verfügen, wir dürfen nur nicht vergessen, dass wir selbst der Patient sind, um den wir uns besonders kümmern sollten.

Die OGKA startet mit September einerseits ins neue Ausbildungsjahr, andererseits schließt sie das alte Semester teilweise erst $a b$, nachdem viele Praktikumskurse auf
Herbst verschoben werden mussten. So einen dicht gedrängten Terminkalender hatte die OGKA wohl noch nie, aber wir freuen uns auf viele spannende Kurse und wissenshungrige Teilnehmer diverser Ausbildungen, Spezialkurse und Praktika. Auch die Vorlesung „Einführung in die Akupunktur" an der MedUni Graz geht in eine neue Runde, wir freuen uns sehr auf unsere Hörer, die neugierig genug sind, über den Tellerrand zu blicken.

Nachdem es für jeden, der beschließt, eine Ausbildung zu beginnen, essenziell ist, diese auch in der vorgegebenen Zeit abschließen zu können, haben wir die OGKA-Ausbildungsgarantie für unsere Theoriekurse entwickelt. Was soviel heißt wie, wenn ein Theoriekurs coronabedingt nicht live stattfinden kann, wird er online abgehalten. Somit gibt es deutlich weniger Kursverschiebungen und man kann damit rechnen, dass der Kurs, den man gebucht hat, stattfindet, in welcher Form auch immer.

Online-Unterricht hat mittlerweile (und oft notgedrungen) keine abschreckende Wirkung mehr, im Gegenteil, wir haben begonnen, uns damit anzufreunden und auch die Vorteile zu sehen. So hat unsere Online-Sommerakademie sehr guten Anklang gefunden und motiviert uns, unser Kursangebot auch online auszubauen.

Im Zuge dessen wird im Herbst auch unsere Homepage in neuem Glanz erstrahlen, seien Sie gespannt!

Ihre

O. Krammer-Pojer

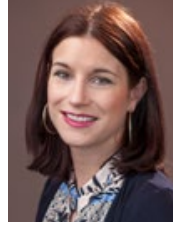

Dr. med. univ. Olivia Krammer-Pojer

Präsidentin der Österreichischen Gesellschaft für kontrollierte Akupunktur und TCM (OGKA)

Glacisstraße 7, A-8010 Graz

Tel. $+43316 / 374050$

E-Mail: office@ogka.at, Internet:www.ogka.at 